\title{
Estimates of Classes of Generalized Special Functions and Their Application in the Fractional $(k, s)$-Calculus Theory
}

\author{
S. Chandak $\mathbb{D},{ }^{1}$ D. L. Suthar $\mathbb{D}^{2},{ }^{2}$ S. AL-Omari $\mathbb{D}^{3}{ }^{3}$ and S. Gulyaz-Ozyurt $\mathbb{D}^{4}$ \\ ${ }^{1}$ Science and Humanities Department, L.D. College of Engineering, Ahmedabad 380015, India \\ ${ }^{2}$ Department of Mathematics, Wollo University, P.O. Box 1145, Dessie, Ethiopia \\ ${ }^{3}$ Department of Physics and Applied Sciences, Al-Balqa Applied University, Amman, Jordan \\ ${ }^{4}$ Department of Mathematics, Sivas Cumhuriyet University, Sivas, Turkey
}

Correspondence should be addressed to D. L. Suthar; dlsuthar@gmail.com

Received 1 September 2021; Accepted 21 September 2021; Published 6 October 2021

Academic Editor: Andreea Fulga

Copyright (c) 2021 S. Chandak et al. This is an open access article distributed under the Creative Commons Attribution License, which permits unrestricted use, distribution, and reproduction in any medium, provided the original work is properly cited.

In this article, we aim to develop new $(k, s)$-fractional integral and differential operators containing $S$-functions as kernels in a form of generalized $k$-Mittag-Leffer functions. We also set up various properties of such operators. Furthermore, we consider a variety of implications of the major outcomes that will be very useful in the implementation of scientific, engineering, and technical problems.

\section{Introduction and Preliminaries}

More focus has been given in recent years to the development of fractional calculus applications. The fractional calculus is very important in the development of integration and differentiation with the fractional calculus powers of real numbers or complex numbers (for example, integral and differential operators). The properties and application of the fractional calculus operator are described by $[1,2]$. For more modern fractional calculus developments, the reader can refer to [3-7]. Some new results for $\psi$-Hilfer fractional pantograph-type differential equation depending on $\psi$-Riemann-Liouville integral are studied by Foukrach et al. [8]. In the frame of fractional derivatives, Alqahtani et al. [9] studied nonlinear $F$-contractions on $b$-metric spaces and differential equations with Mittag-Leffler kernel. Many scholars have computed numerous fractional integral inequalities containing the various fractional integration and differentiation operators over the past few years (see $[10,11])$. The $k$ symbols are well known from a number of sources related to the measurement of finite differences (see $[12,13])$. In the literature, $k$-fractional integral operators have recently been considered by different scholars.
For this function, we begin the literature with the following properties. The Pochhammer $k$-symbols and $k$-gamma function were introduced by Diaz and Pariguan (see [14])

$$
\begin{aligned}
(\zeta)_{n, k}= \begin{cases}1, & (n=0, \zeta \in \mathbb{C}), \\
\zeta(\zeta+k) \cdots(\zeta+(n-1) k), & (n \in \mathbb{N}, \zeta \in \mathbb{C}, k>0),\end{cases} \\
\Gamma_{k}(\rho)=\int_{0}^{\infty} z^{\rho-1} e^{-z^{k} / k} d z, \quad \rho \in \mathbb{C}, \mathfrak{R}(\rho)>0 .
\end{aligned}
$$

In the same paper, they spell out the relations

$$
\begin{array}{r}
\Gamma_{k}(\rho+k)=\rho \Gamma_{k}(\rho), \\
\Gamma_{k}(\rho)=(k)^{(\rho / k)-1} \Gamma\left(\frac{\rho}{k}\right) .
\end{array}
$$

The $k$-fractional integral is develop by [15] as

$$
I_{k}^{\varsigma}(f(x))=\frac{1}{k \Gamma_{k}(\varsigma)} \int_{0}^{x}(x-z)^{(\varsigma / k)-1} f(z) d z
$$


When we choose $k=1$, then $I_{k}^{\varsigma}(f(x))$ shows the result of the Riemann-Liouville (R-L) fractional integration formula. We have

$$
I^{\varsigma}(f(x))=\frac{1}{\Gamma(\varsigma)} \int_{0}^{x}(x-z)^{\varsigma-1} f(z) d z
$$

Formulas for $k$-fractional integral are developed by [15] as

$$
\begin{gathered}
I_{k}^{\varsigma}\left(x^{(v / k)-1}\right)=\frac{\Gamma_{k}(v)}{\Gamma_{k}(\rho+v)} x^{((\rho / k)+(v / k))-1}, \\
I_{k}^{\varsigma}\left((x-u)^{(v / k)-1}\right)=\frac{\Gamma_{k}(v)}{\Gamma_{k}(\rho+v)}(x-u)^{((\rho / k)+(v / k))-1} .
\end{gathered}
$$

The R-L $(k, s)$-fractional integral of order $\varsigma>0$ was elucidated by [16].

$$
{ }_{k}^{s} I_{a}^{\varsigma} f(x)=\frac{(s+1)^{1-(\varsigma / k)}}{k \Gamma_{k}(\varsigma)} \int_{a}^{x}\left(x^{s+1}-z^{s+1}\right)^{(\varsigma / k)-1} z^{s} f(z) d z,
$$

where $x \in[a, b], k>0$ and $s \in \Re \backslash\{-1\}$. In the same paper, they defined the following result:

$$
\begin{aligned}
{ }_{k}^{s} I_{a}^{\varsigma} & {\left[\left(z^{s+1}-a^{s+1}\right)^{\theta / k}-1\right] } \\
& =\frac{\Gamma_{k}(\theta)}{(s+1)^{(\varsigma / k)-1} \Gamma_{k}(\theta+\varsigma)}\left(x^{s+1}-a^{s+1}\right)^{((\theta+\varsigma) / k)-1} .
\end{aligned}
$$

In recent years, the applications of the fractional calculus are given by the researchers (see $[17,18]$ ). By using generalized $k$-fractional integrals, Gruss-type integral inequalities for generalized R-L $k$-fractional integrals, and $(k, s)$-R-L fractional integral inequalities for continuous random variables, analytical properties of $(k, s)$-RiemannLiouville fractional integral several researchers have also provided such results including Hermite-Hadamard-type inequalities by using the definition of $(k, s)$-fractional integrals [19-23].

The $S$-function is given by [24]

$$
\begin{aligned}
S_{(l, m)}^{(\varsigma, v, \sigma, \xi, k)} & \left(p_{1}, \cdots, p_{l} ; q_{1}, \cdots, q_{m} ; x\right) \\
& =\sum_{n=0}^{\infty} \frac{\left(p_{1}\right)_{n} \cdots\left(p_{l}\right)_{n}(\sigma)_{n \xi, k} x^{n}}{\left(q_{1}\right)_{n} \cdots\left(q_{m}\right)_{n} \Gamma_{k}(n \varsigma+v) n !},
\end{aligned}
$$

$k \in \Re ; \varsigma, v, \sigma, \xi \in \mathbb{C} ; \mathfrak{R}(\varsigma)>0, p_{i}(i=1,2, \cdots, l), q_{j}(j=1,2, \cdots$, $m), \mathfrak{R}(\varsigma)>k \Re(\xi)$, and $l<m+1$.

The Pochhammer symbol $(\zeta)_{n},(\zeta \in \mathbb{C}, n \in \mathbb{N})$ defined in terms of gamma function as (also see [7])

$$
(\zeta)_{n}= \begin{cases}1, & (n=0, \zeta \in \mathbb{C}) \\ \zeta(\zeta+1) \cdots(\zeta+n-1), & (n \in \mathbb{N}, \zeta \in \mathbb{C})\end{cases}
$$

For some more result on $S$-function, the reader can refer to [25-29].

\subsection{Special Cases}

(i) Choosing $l=0=m$ in equation (9), it becomes generalized $k$-Mittag-Leffler function, defined by Saxena et al. [30].

$$
S_{(0,0)}^{(\varsigma, v, \xi, k)}(-;-; x)=\sum_{n=0}^{\infty} \frac{(\sigma)_{n \xi, k} x^{n}}{\Gamma_{k}(n \varsigma+v) n !}=E_{k, \varsigma, v}^{\sigma, \xi}(x)
$$

where $\mathfrak{R}((\varsigma / k)-\xi)>l-m$.

(ii) For $\xi=m$, equation (9) yields

$S_{(l, m)}^{(\varsigma, v, \sigma, q, k)}\left(p_{1}, \cdots, p_{l} ; q_{1}, \cdots, q_{m} ; x\right)=\sum_{n=0}^{\infty} \frac{\left(p_{1}\right)_{n} \cdots\left(p_{l}\right)_{n}(\sigma)_{n m, k} x^{n}}{\left(q_{1}\right)_{n} \cdots\left(q_{m}\right)_{n} \Gamma_{k}(n \varsigma+v) n !}$,

where $\mathfrak{R}(\varsigma)>k l$.

(iii) When $\xi=1$, equation (9) yields

$S_{(l, m)}^{(\varsigma, v, \sigma, 1, k)}\left(p_{1}, \cdots, p_{l} ; q_{1}, \cdots, q_{m} ; x\right)=\sum_{n=0}^{\infty} \frac{\left(p_{1}\right)_{n} \cdots\left(p_{l}\right)_{n}(\sigma)_{n, k} x^{n}}{\left(q_{1}\right)_{n} \cdots\left(q_{m}\right)_{n} \Gamma_{k}(n \varsigma+v) n !}$

$$
=S_{(l, m)}^{(\varsigma, v, \sigma, k)}\left(p_{1}, \cdots, p_{l} ; q_{1}, \cdots, q_{m} ; x\right),
$$

where $\mathfrak{R}(\varsigma)>k l$.

(iv) For $k=1$, equation (14) yields $K$-function defined by Sharma [31]

$$
\begin{gathered}
S_{(l, m)}^{(\varsigma, v, \sigma, 1,1)}\left(p_{1}, \cdots, p_{l} ; q_{1}, \cdots, q_{m} ; x\right)=\sum_{n=0}^{\infty} \frac{\left(p_{1}\right)_{n} \cdots\left(p_{l}\right)_{n}(\sigma)_{n} x^{n}}{\left(q_{1}\right)_{n} \cdots\left(q_{m}\right)_{n} \sigma(n \varsigma+v) n !} \\
=K_{(l, m)}^{(\varsigma, v, \sigma)}\left(p_{1}, \cdots, p_{l} ; q_{1}, \cdots, q_{m} ; x\right),
\end{gathered}
$$

where $\mathfrak{R}(\varsigma)>l-m$.

(v) When $\sigma=1$ (equation (16)), it reduces to generalized $M$-series defined by [32] (see also [33])

$$
S_{(l, m)}^{(\varsigma, v, 1,1,1)}\left(p_{1}, \cdots, p_{l} ; q_{1}, \cdots, q_{m} ; x\right)=\sum_{n=0}^{\infty} \frac{\left(p_{1}\right)_{n} \cdots\left(p_{l}\right)_{n} x^{n}}{\left(q_{1}\right)_{n} \cdots\left(q_{m}\right)_{n} \Gamma(n \varsigma+v) n !}
$$

$$
=M_{(l, m)}^{(\varsigma, v)}\left(p_{1}, \cdots, p_{l} ; q_{1}, \cdots, q_{m} ; x\right),
$$

where $\mathfrak{R}(\varsigma)>l-m-1$.

(vi) When $p=q=0$ (equation (18)), it reduces to generalized Mittag-Leffler function, defined by [34] 


$$
S_{(0,0)}^{(\zeta,, 1,1,1)}(-;-; x)=\sum_{n=0}^{\infty} \frac{x^{n}}{\Gamma(n \varsigma+v) n !}=E_{\zeta, v}(x),
$$

where $\varsigma, v \in \mathbb{C}, \mathfrak{R}(\varsigma)>0, \mathfrak{R}(v)>0$.

\section{2. $(k, s)$-Fractional Integrals and Differentials of $S$-Function}

In this section, we develop $(k, s)$-fractional integration and differentiation operators containing $S$-function as its kernel. Also, we study $(k, s)$-fractional calculus; we define integral operators in terms of $(k, s)$ as follows.

Definition 1. If $k>0$ and $\varsigma, v, \sigma, \xi, \zeta, \tau \in \mathbb{C}, \mathfrak{R}(\varsigma)>0, \Re(v)$ $>0, \mathfrak{R}(\sigma)>0$, then

$$
\begin{aligned}
\left({ }_{k}^{s} \varepsilon_{a+;, \sigma, v}^{\tau ; \sigma, \xi} f\right)(x)= & \frac{1}{k} \int_{a}^{x}\left(x^{s+1}-z^{s+1}\right)^{(v / k)-1} \\
& \cdot S_{(l, m)}^{(\zeta, v, \sigma, \xi, k)}\left(\tau\left(x^{s+1}-z^{s+1}\right)^{\varsigma / k}\right) z^{s} f(z) d z,
\end{aligned}
$$

where $x>\rho$. Substituting $s=0$, then (20) reduces to the operator

$$
\left({ }_{k} \varepsilon_{a+; \zeta, v}^{\tau ; \sigma} f\right)(x)=\frac{1}{k} \int_{a}^{x}(x-z)^{(v / k)-1} S_{(l, m)}^{(\varsigma, v, \sigma, \xi, k)}\left(\tau(x-z)^{\zeta / k}\right) f(z) d z .
$$

In particular, the integral operator in (21) decreases to the well-known R-L fractional integral operator defined as $\tau=0$ and $k=1$.

$$
\left(I_{a+}^{\varsigma} f\right)(x)=\frac{1}{\varsigma} \int_{a}^{x}(x-z)^{v-1} f(z) d z .
$$

The integral operator is described as ${ }_{k}^{s} I_{a+}^{\varsigma}$ and ${ }_{k}^{s} I_{a-}^{\varsigma}$, and also, $(k, s)$-fractional order left side and right side fractional differential operators are described as $(k, s)-D_{\rho+, k}^{\varsigma}$ and $D_{\rho-, k}^{\varsigma}$. Also, we used left- and right-sided R-L $(k, s)$-fractional integral operators ${ }_{k}^{s} I_{a^{+}}^{\zeta}$ and ${ }_{k}^{s} I_{a^{-}}^{\varsigma}$. Similarly, the left- and rightsided R-L $(k, s)$-fractional differential operators are ${ }_{k}^{s} D_{a+, k}^{\varsigma}$ and ${ }_{k}^{s} D_{a-}^{\varsigma}$, respectively. By using the Lebesgue measurable integral of a real or complex valued function, we can describe both R-L $(k, s)$-fractional integral operators. The Lebesgue measurable integral of a valued function that is denoted and defined as real or complex form

$$
L(\rho, v)=\left\{f:\|\psi\|_{1}=\int_{\rho}^{v}|\psi(\xi)| d \xi<\infty ; \psi \in L(\rho, v)\right\} .
$$

Definition 2. For $\psi(x) \in L(\rho, v), \varsigma \in \mathbb{C}, \mathfrak{R}(\varsigma)>0, k>0$, then we define the R-L left-sided $(k, s)$-fractional integral operator of order $\varsigma$ as

$$
\begin{aligned}
{ }_{a, k}^{s} D_{x}^{-\varsigma} f(x) & ={ }_{a, k}^{s} I_{x}^{\varsigma} f(x)={ }_{k}^{s} I_{a+}^{\varsigma} f(x)=\left({ }_{k}^{s} I_{a+}^{\varsigma} f\right)(x) \\
& =\frac{(s+1)^{1-(\varsigma / k)}}{k \Gamma_{k}(\varsigma)} \int_{a}^{x}\left(x^{s+1}-z^{s+1}\right)^{(\varsigma / k)-1} z^{s} f(z) d z(x>a) .
\end{aligned}
$$

The R-L right-sided $(k, s)$-fractional integral operator of order $\varsigma$ is defined as

$$
\begin{aligned}
{ }_{\rho, k}^{s} D_{v}^{-\varsigma} f(x) & ={ }_{\rho, k}^{s} I_{v}^{\varsigma} f(x)={ }_{k}^{s} I_{a-}^{\varsigma} f(x)=\left({ }_{k}^{s} I_{a+}^{\varsigma} f\right)(x) \\
& =\frac{(s+1)^{1-(\varsigma / k)}}{k \Gamma_{k}(\varsigma)} \int_{x}^{v}\left(x^{s+1}-z^{s+1}\right)^{(\varsigma / k)-1} z^{s} f(z) d z(x<v) .
\end{aligned}
$$

Definition 3. For $k>0 ; s \in \mathfrak{R}\{-1\} ; \varsigma \in \mathbb{C}, \mathfrak{R}(\varsigma)>0$ and $n=$ $[\mathfrak{R}(\varsigma)]+1$, then the R-L left- and right-sided $(k, s)$-fractional differential operators are defined as

$$
\begin{aligned}
& \left({ }_{k}^{s} D_{a+}^{\varsigma} f\right)(x)=\left[\left(\frac{1}{x^{s}} \frac{d}{d x}\right)^{n}\right]\left(k^{n s} I_{a+}^{n k-\varsigma} f\right)(x), \\
& \left({ }_{k}^{s} D_{a-}^{\varsigma} f\right)(x)=\left[\left(-\frac{1}{x^{s}} \frac{d}{d x}\right)^{n}\right]\left(k^{n s} I_{a-}^{n k-\varsigma} f\right)(x),
\end{aligned}
$$

respectively. Substituting $k=1$ and $s=0$, then the R-L left- and right-sided $(k, s)$-fractional integrals and derivatives will reduce to the well-known R-L left-side and rightside fractional integrals and derivatives; see $[35,36]$.

Definition 4. The generalized $(k, s)$-fractional derivative operator is denoted by ${ }_{k}^{s} D_{a+}^{\varsigma, v}$ where $\varsigma$ is the order such that $0<\varsigma<1$ and $0<v<1$ is defined as

$$
\left({ }_{k}^{s} D_{a+}^{\zeta, v} f\right)(x)=\left[{ }_{k}^{s} I_{\rho+}^{\zeta(k-v)}\left(\frac{1}{x^{s}} \frac{d}{d x}\right)\left(k_{k}^{s} I_{a+}^{(1-v)(k-\varsigma)} f\right)\right](x) .
$$

Obviously, when $v=0$, then (27) reduces to the R-L $(k, s)$-fractional derivative operators ${ }_{k}^{s} D_{a+}^{\varsigma}(24)$.

Lemma 5. For $k>0$, the following result for $(k, s)$-fractional derivative operator $D_{a+, k}^{\varsigma, v}$ defined in (27) holds true:

$$
\begin{aligned}
& \left({ }_{k}^{s} D_{a+, k}^{\varsigma, v}\left[\left(z^{s+1}-a^{s+1}\right)^{(\theta / k)-1}\right]\right)(x) \\
& \quad=\frac{\Gamma_{k}(\theta)}{(s+1)^{-\varsigma / k} \Gamma_{k}(\theta-\varsigma)}\left(x^{s+1}-a^{s+1}\right)^{((\theta-\varsigma) / k)-1},
\end{aligned}
$$

with $x>\rho, 0<\varsigma<1,0<v<1$, and $\mathfrak{R}(\theta)>0$.

Proof. We obtain from equation (8)

$$
\begin{aligned}
& \left({ }_{k}^{s} I_{a+}^{(1-v)(k-\varsigma)}\left[\left(z^{s+1}-a^{s+1}\right)^{(\theta / k)-1}\right]\right)(x) \\
& \quad=\frac{\Gamma_{k}(\theta)}{(s+1)^{((1-v)(k-\varsigma)) / k-1} \Gamma_{k}((1-v)(k-\varsigma)+\theta)} \\
& \cdot\left(x^{s+1}-a^{s+1}\right)^{(((1-v)(k-\varsigma)+\theta) / k)-1},
\end{aligned}
$$




$$
\begin{aligned}
\frac{1}{x^{s}} \frac{d}{d x} & \left({ }_{k}^{s} I_{a+}^{(1-v)(k-\varsigma)}\left[\left(z^{s+1}-a^{s+1}\right)^{(\theta / k)-1}\right]\right)(x) \\
= & \frac{[(1-v)(k-\varsigma)+\theta-k] \Gamma_{k}(\theta)}{k(s+1)^{(((1-v)(k-\varsigma)) / k)-1} \Gamma_{k}((1-v)(k-\varsigma)+\theta)} \\
& \cdot\left(x^{s+1}-a^{s+1}\right)^{(((1-v)(k-\varsigma)+\theta) / k)-2},
\end{aligned}
$$

which by applying the relation given by (2) yields

$$
\begin{aligned}
\left({ }_{k}^{s} D_{a+, k}^{\varsigma, v}\left[\left(z^{s+1}-a^{s+1}\right)^{(\theta / k)-1}\right]\right)(x) \\
=\frac{\Gamma_{k}(\theta)}{\Gamma_{k}((1-v)(k-\varsigma)+\theta-k)} \\
\quad \cdot\left({ }_{k}^{s} I_{a+}^{v(k-\varsigma)}\left[\left(z^{s+1}-a^{s+1}\right)^{(((1-v)(k-\varsigma)+\theta) / k)-2}\right]\right)(x) \\
=\frac{\Gamma_{k}(\theta)}{(s+1)^{((v(k-\varsigma)+(1-v)(k-\varsigma)) / k)-1} \Gamma_{k}((1-v)(k-\varsigma)+\theta-k)} \\
\quad \times \frac{\Gamma_{k}((1-v)(k-\varsigma)+\theta-k)}{\Gamma_{k}(v(k-\varsigma)+(1-v)(k-\varsigma)+\theta-k)} \\
\cdot\left(x^{s+1}-a^{s+1}\right)((\theta-\varsigma) / k)-1 \\
=\frac{\Gamma_{k}(\theta)}{(s+1)^{-v / k} \Gamma_{k}(\theta-v)}\left(x^{s+1}-a^{s+1}\right)^{(\theta-\varsigma) / k-1},
\end{aligned}
$$

which is the desired proof.

Theorem 6. For $k>0$, the following result always holds true:

$$
\begin{gathered}
\left(\frac{1}{x^{s}} \frac{d}{d x}\right)^{\alpha}\left[\left(x^{s+1}-a^{s+1}\right)^{(v / k)-1} S_{(l, m)}^{(\varsigma, v, \xi \xi, k)}\left(\tau\left(x^{s+1}-a^{s+1}\right)^{\varsigma / k}\right)\right] \\
=\frac{(s+1)^{\alpha}\left(x^{s+1}-a^{s+1}\right)^{(v / k)-\alpha-1}}{k^{\alpha}} \\
\cdot S_{(l, m)}^{(\zeta, v-\alpha k, \sigma, \xi, k)}\left(\tau\left(x^{s+1}-a^{s+1}\right)^{\varsigma / k}\right),
\end{gathered}
$$

$\sigma)>0$.

where $s \in \mathfrak{R} \backslash[-1], \varsigma, v, \sigma, \xi \in \mathbb{C}, \mathfrak{R}(v)>0, \mathfrak{R}(\varsigma)>0, \mathfrak{R}($

Proof. The proof is obvious by applying $\left(1 / x^{s} d / d x\right)^{\alpha}$ where $\alpha=1,2$,

Corollary 7. Choosing $l=0=m$ in equation (32), it becomes generalized $k$-Mittag-Leffler function and we get a result earlier given by Nisar et al. [37].

Corollary 8. Choosing $\xi=m$ in equation (32) and using equation (12), we get

$$
\begin{gathered}
\left(\frac{1}{x^{s}} \frac{d}{d x}\right)^{\alpha}\left[\left(x^{s+1}-a^{s+1}\right)^{(v / k)-1} S_{(l, m)}^{(\zeta,, \sigma, m, k)}\left(\tau\left(x^{s+1}-a^{s+1}\right)^{\varsigma / k}\right)\right] \\
=\frac{(s+1)^{\alpha}\left(x^{s+1}-a^{s+1}\right)^{(v / k)-\alpha-1}}{k^{\alpha}} \\
\cdot S_{(l, m)}^{(\zeta, v-\alpha k, \sigma, m, k)}\left(\tau\left(x^{s+1}-a^{s+1}\right)^{\zeta / k}\right) .
\end{gathered}
$$

Corollary 9. Choosing $\xi=1$ in equation (32) and using (14) yield

$$
\begin{gathered}
\left(\frac{1}{x^{s}} \frac{d}{d x}\right)^{\alpha}\left[\left(x^{s+1}-a^{s+1}\right)^{(v / k)-1} S_{(l, m)}^{(\varsigma, v, \sigma, 1, k)}\left(\tau\left(x^{s+1}-a^{s+1}\right)^{\varsigma / k}\right)\right] \\
=\frac{(s+1)^{\alpha}\left(x^{s+1}-a^{s+1}\right)^{(v / k)-\alpha-1}}{k^{\alpha}} \\
\cdot S_{(l, m)}^{(\zeta, v-\alpha k, \sigma, k)}\left(\tau\left(x^{s+1}-a^{s+1}\right)^{\varsigma / k}\right) .
\end{gathered}
$$

Corollary 10. Choosing $k=1$ in equation (34) then S-function converted in to K-function and using (16) yield

$$
\begin{aligned}
\left(\frac{1}{x^{s}} \frac{d}{d x}\right)^{\alpha} & {\left[\left(x^{s+1}-a^{s+1}\right)^{v-1} S_{(l, m)}^{(\varsigma, v, \sigma, 1,1)}\left(\tau\left(x^{s+1}-a^{s+1}\right)^{\varsigma}\right)\right] } \\
& =(s+1)^{\alpha}\left(x^{s+1}-a^{s+1}\right)^{v-\alpha-1} K_{(l, m)}^{(\varsigma, v-\alpha, \sigma)}\left(\tau\left(x^{s+1}-a^{s+1}\right)^{\varsigma}\right) .
\end{aligned}
$$

Corollary 11. Choosing $\sigma=1$ in equation (35) and using equation (16), it becomes generalized M-series defined by Sharma and Jain [32]

$$
\begin{aligned}
\left(\frac{1}{x^{s}} \frac{d}{d x}\right)^{m \alpha} & {\left[\left(x^{s+1}-a^{s+1}\right)^{v-1} S_{(l, m)}^{(\zeta, v, 1,1)}\left(\tau\left(x^{s+1}-a^{s+1}\right)^{\varsigma}\right)\right] } \\
& =(s+1)^{\alpha}\left(x^{s+1}-a^{s+1}\right)^{v-\alpha-1} M_{(l, m)}^{(\zeta, v-\alpha)}\left(\tau\left(x^{s+1}-a^{s+1}\right)^{\varsigma}\right) .
\end{aligned}
$$

Corollary 12. Choosing $l=0=m$ in equation (36), it becomes generalized Mittag-Leffler function [34]

$$
\begin{aligned}
\left(\frac{1}{x^{s}} \frac{d}{d x}\right)^{\alpha} & {\left[\left(x^{s+1}-a^{s+1}\right)^{v-1} S_{(0,0)}^{(\varsigma, v, l, 1)}\left(\tau\left(x^{s+1}-a^{s+1}\right)^{\varsigma}\right)\right] } \\
& =(s+1)^{\alpha}\left(x^{s+1}-a^{s+1}\right)^{v-\alpha-1} E_{(\varsigma, v-\alpha)}\left(\tau\left(x^{s+1}-a^{s+1}\right)^{\varsigma}\right) .
\end{aligned}
$$

Theorem 13. Suppose $k>0, x>a\left(a \in \mathfrak{R}_{+}=[0, \infty)\right)$ and $\varsigma$, $\nu, \sigma, \tau \in \mathbb{C}, \mathfrak{R}(v), \mathfrak{R}(\varsigma)>0, \mathfrak{R}(\sigma)>0, \mathfrak{R}(\varsigma)>0$, then

$$
\begin{gathered}
\left({ }_{k}^{s} I_{a+}^{\varsigma}\left[\left(z^{s+1}-a^{s+1}\right)^{(v / k)-1} S_{(l, m)}^{(\varsigma, v, \sigma, \xi, k)}\left(\tau\left(z^{s+1}-a^{s+1}\right)^{\varsigma / k}\right)\right]\right)(x) \\
=\frac{\left(x^{s+1}-a^{s+1}\right)^{((v+\varsigma) / k)-1}}{(s+1)^{\varsigma / k}} S_{(l, m)}^{(\zeta, v+, \sigma, \xi, k)}\left(\tau\left(x^{s+1}-a^{s+1}\right)^{\varsigma / k}\right), \\
\left({ }_{k}^{s} D_{a+}^{\varsigma}\left[\left(z^{s+1}-a^{s+1}\right)^{(v / k)-1} S_{(l, m)}^{(\zeta, v, \sigma, \xi, k)}\left(\tau\left(z^{s+1}-a^{s+1}\right)^{\varsigma / k}\right)\right]\right)(x) \\
=\frac{\left(x^{s+1}-a^{s+1}\right)^{((v+\varsigma) / k)-1}}{(s+1)^{\zeta / k}} S_{(l, m)}^{(\varsigma, v-\zeta, \sigma, \xi, k)}\left(\tau\left(x^{s+1}-a^{s+1}\right)^{\varsigma / k}\right),
\end{gathered}
$$




$$
\begin{gathered}
\left({ }_{k}^{s} D_{a+}^{\varsigma, v}\left[\left(z^{s+1}-a^{s+1}\right)^{(v / k)-1} S_{(l, m)}^{(\varsigma, v, \sigma, \xi, k)}\left(\tau\left(z^{s+1}-a^{s+1}\right)^{\zeta / k}\right)\right]\right)(x) \\
=\frac{\left(x^{s+1}-a^{s+1}\right)^{((v+\zeta) / k)-1}}{(s+1)^{-\zeta / k}} S_{(,, m)}^{(\varsigma, v-\zeta, \sigma, \xi, k)}\left(\tau\left(x^{s+1}-a^{s+1}\right)^{\varsigma / k}\right) .
\end{gathered}
$$

Proof. By using equation (7), we get

$$
\begin{aligned}
& \left({ }_{k}^{s} I_{a+}^{\varsigma}\left[\left(z^{s+1}-a^{s+1}\right)^{(v / k)-1} S_{(l, m)}^{(\zeta,, \sigma, \xi)}\left(\tau\left(z^{s+1}-a^{s+1}\right)^{\varsigma / k}\right)\right]\right)(x) \\
& =\frac{(s+1)^{1-(\varsigma / k)}}{k \Gamma_{k}(\varsigma)} \int_{a}^{x} \frac{\left(z^{s+1}-a^{s+1}\right)^{(v / k)-1} S_{(l, m)}^{(\zeta, \sigma, \xi, k)}\left(\tau\left(z^{s+1}-a^{s+1}\right)^{\varsigma / k}\right) z^{s}}{\left(x^{s+1}-a^{s+1}\right)^{1-(\varsigma / k)}} d z \\
& =\frac{(s+1)^{1-(\varsigma / k)}}{k \Gamma_{k}(\varsigma)} \sum_{n=0}^{\infty} \frac{\left(p_{1}\right)_{n} \cdots\left(p_{l}\right)_{n}(\sigma)_{n \xi \xi, k} \tau^{n}}{\left(q_{1}\right)_{n} \cdots\left(q_{m}\right)_{n} \Gamma_{k}(n \varsigma+v) n !} \\
& \quad \times \int_{a}^{x}\left(z^{s+1}-a^{s+1}\right)^{((v+\varsigma n) / k)-1}\left(x^{s+1}-a^{s+1}\right)^{(\varsigma / k)-1} z^{s} d z .
\end{aligned}
$$

Substituting $z^{s+1}=a^{s+1}+y\left(x^{s+1}-a^{s+1}\right)$, this implies $z^{s}$ $d z=\left(\left(x^{s+1}-a^{s+1}\right) /(s+1)\right) d y$; we get

$$
\begin{aligned}
& \left({ }_{k}^{s} I_{a^{+}}^{\varsigma}\left[\left(z^{s+1}-a^{s+1}\right)^{(v / k)-1} S_{(l, m)}^{(s, v, \xi \xi, k)}\left(\tau\left(z^{s+1}-a^{s+1}\right)^{\varsigma / k}\right)\right]\right)(x) \\
& =\frac{(s+1)^{1-(\varsigma / k)}}{(s+1) k \Gamma_{k}(\varsigma)} \sum_{n=0}^{\infty} \frac{\left(p_{1}\right)_{n} \cdots\left(p_{l}\right)_{n}(\sigma)_{n \xi, k} \tau^{n}}{\left(q_{1}\right)_{n} \cdots\left(q_{m}\right)_{n} \Gamma_{k}(n \varsigma+v) n !} \\
& \times \int_{0}^{1}\left(x^{s+1}-a^{s+1}\right)^{((v+\varsigma n+\varsigma) / k)-1}(1-y)^{(\zeta / k)-1} y^{((v+\rho n) / k)-1} d y \\
& =\frac{\left(x^{s+1}-a^{s+1}\right)^{((v+\varsigma) / k)-1}}{(s+1)^{\varsigma / k} k \Gamma_{k}(\varsigma)} \\
& \cdot \sum_{n=0}^{\infty} \frac{\left(p_{1}\right)_{n} \cdots\left(p_{l}\right)_{n}(\sigma)_{n \xi, k}\left(\tau\left(x^{s+1}-a^{s+1}\right)^{\varsigma / k}\right)^{n} \Gamma_{k}(n \varsigma+v) \Gamma_{k}(\varsigma)}{\left(q_{1}\right)_{n} \cdots\left(q_{m}\right)_{n} \Gamma_{k}(n \varsigma+v) \Gamma_{k}(\varsigma+v+n \varsigma) \quad n !} \\
& =\frac{\left(x^{s+1}-a^{s+1}\right)^{((v+\zeta) / k)-1}}{(s+1)^{\varsigma / k}} \sum_{n=0}^{\infty} \frac{\left(p_{1}\right)_{n} \cdots\left(p_{l}\right)_{n}(\sigma)_{n \xi, k}\left(\tau\left(x^{s+1}-a^{s+1}\right)^{\varsigma / k}\right)^{n}}{\left(q_{1}\right)_{n} \cdots\left(q_{m}\right)_{n} \Gamma_{k}(\varsigma+v+n \varsigma) n !} \\
& =\frac{\left(x^{s+1}-a^{s+1}\right)^{((v+\zeta) / k)-1}}{(s+1)^{\zeta / k}} S_{(l, m)}^{(\zeta, v+\zeta, \xi, \xi k)}\left(\tau\left(z^{s+1}-a^{s+1}\right)^{\zeta / k}\right) \text {. }
\end{aligned}
$$

This completes the proof of (38).

Now, to prove (39),

$$
\begin{aligned}
& \left({ }_{k}^{s} D_{a+}^{\varsigma}\left[\left(z^{s+1}-a^{s+1}\right)^{(v / k)-1} S_{(l, m)}^{(\varsigma,, \sigma, \xi, k)}\left(\tau\left(z^{s+1}-a^{s+1}\right)^{\varsigma / k}\right)\right]\right)(x) \\
& =\left(\frac{1}{x^{s}} \frac{d}{d x}\right)^{n}\left(k ^ { n s } I _ { a + } ^ { n k - \varsigma } \left[\left(z^{s+1}-a^{s+1}\right)^{(v / k)-1} S_{(l, m)}^{(\zeta,, \sigma, \xi, k)}\right.\right. \\
& \left.\left.\cdot\left(\tau\left(z^{s+1}-a^{s+1}\right)^{\varsigma / k}\right)\right]\right)(x),
\end{aligned}
$$

and using (38), this take the following form:

$$
\begin{aligned}
& \left({ }_{k}^{s} D_{a+}^{\varsigma}\left[\left(z^{s+1}-a^{s+1}\right)^{(v / k)-1} S_{(l, m)}^{(\varsigma, v, \sigma, \xi, k)}\left(\tau\left(z^{s+1}-a^{s+1}\right)^{\zeta / k}\right)\right]\right)(x) \\
& =k^{n}\left(\frac{1}{x^{s}} \frac{d}{d x}\right)^{n}\left(( s + 1 ) ^ { - ( \varsigma / k ) - n } \left[\left(x^{s+1}-a^{s+1}\right)^{((v-\varsigma) / k)+n-1}\right.\right. \\
& \left.\left.\cdot S_{(l, m)}^{(\varsigma, v-\varsigma+n k, \sigma, \xi, k)}\left(\tau\left(x^{s+1}-a^{s+1}\right)^{\varsigma / k}\right)\right]\right) .
\end{aligned}
$$

\section{Applying (32), we have}

$$
\begin{aligned}
& \left({ }_{k}^{s} D_{a^{+}}^{\varsigma}\left[\left(z^{s+1}-a^{s+1}\right)^{(v / k)-1} S_{(l, m)}^{(\varsigma, v, \sigma, \xi, k)}\left(\tau\left(z^{s+1}-a^{s+1}\right)^{\varsigma / k}\right)\right]\right)(x) \\
& =\left[(s+1)^{-\varsigma / k}\left(x^{s+1}-a^{s+1}\right)^{((v-\varsigma) / k)+n-1} S_{(l, m)}^{(\zeta, v-\varsigma+n k, \sigma, \xi, k)}\right. \\
& \left.\quad \cdot\left(\tau\left(x^{s+1}-a^{s+1}\right)^{\varsigma / k}\right)\right] .
\end{aligned}
$$

This completes the desired proof.

Now, to prove (40), we have

$$
\begin{aligned}
& \left({ }_{k}^{s} D_{a+}^{\zeta, v}\left[\left(z^{s+1}-a^{s+1}\right)^{(v / k)-1} S_{(l, m)}^{(\zeta, v, \xi, k)}\left(\tau\left(z^{s+1}-a^{s+1}\right)^{\zeta / k}\right)\right]\right)(x)
\end{aligned}
$$

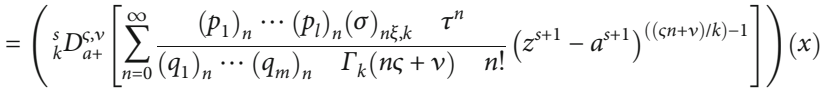

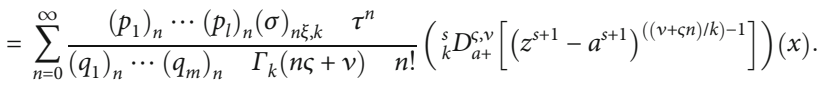

Using (28), we get

$$
\begin{aligned}
& \left({ }_{k}^{s} D_{a+}^{\varsigma}\left[\left(z^{s+1}-a^{s+1}\right)^{(v / k)-1} S_{(l, m)}^{(\varsigma,,, \sigma, \xi, k)}\left(\tau\left(z^{s+1}-a^{s+1}\right)^{\varsigma / k}\right)\right]\right)(x) \\
& =\sum_{n=0}^{\infty} \frac{\left(p_{1}\right)_{n} \cdots\left(p_{l}\right)_{n}(\sigma)_{n \xi, k} \quad \tau^{n}}{\left(q_{1}\right)_{n} \cdots\left(q_{m}\right)_{n}} \Gamma_{k}(n \varsigma+v) \quad n ! \frac{\Gamma_{k}(v+\varsigma n)}{(s+1)^{-\zeta / k} \Gamma_{k}(v+\varsigma n-\varsigma)} \\
& \cdot\left(x^{s+1}-a^{s+1}\right)^{((v+\varsigma n-\varsigma) / k)-1}=\frac{\left(x^{s+1}-a^{s+1}\right)^{((v-\varsigma) / k)-1}}{(s+1)^{-\varsigma / k}} \\
& \cdot \sum_{n=0}^{\infty} \frac{\left(p_{1}\right)_{n} \cdots\left(p_{l}\right)_{n}(\sigma)_{n \xi, k} \quad\left(\tau\left(x^{s+1}-a^{s+1}\right)^{\zeta / k}\right)^{n}}{\left(q_{1}\right)_{n} \cdots\left(q_{m}\right)_{n} \quad \Gamma_{k}(n \varsigma+v-\varsigma) n !} \\
& =\frac{\left(x^{s+1}-a^{s+1}\right)^{((v-\varsigma) / k)-1}}{(s+1)^{-\varsigma / k}} S_{(l, m)}^{(\zeta,-\zeta, \sigma, \xi, k)}\left(\tau\left(x^{s+1}-a^{s+1}\right)^{\zeta / k}\right) \text {, }
\end{aligned}
$$

which completes the desired proof.

Corollary 14. Choosing $p=q=0$ in equations (38), (39), and (40), it reduces to generalized $k$-Mittag-Leffler function and we get a result earlier given by Nisar et al. [37]. 
Corollary 15. Choosing $\xi=q$ in equations (38), (39), and (40) and using equation (12), we get

$$
\begin{aligned}
& \left({ }_{k}^{s} I_{a+}^{\varsigma}\left[\left(z^{s+1}-a^{s+1}\right)^{(v / k)-1} S_{(l, m)}^{(\zeta, v, q, k)}\left(\tau\left(z^{s+1}-a^{s+1}\right)^{\zeta / k}\right)\right]\right)(x) \\
& =\frac{\left(x^{s+1}-a^{s+1}\right)^{((v+\zeta) / k)-1}}{(s+1)^{\zeta / k}} S_{(l, m)}^{(\varsigma, v+\zeta, \sigma, q, k)}\left(\tau\left(x^{s+1}-a^{s+1}\right)^{\varsigma / k}\right), \\
& \left({ }_{k}^{s} D_{a+}^{\varsigma}\left[\left(z^{s+1}-a^{s+1}\right)^{(v / k)-1} S_{(l, m)}^{(\varsigma, v, \sigma, q, k)}\left(\tau\left(z^{s+1}-a^{s+1}\right)^{\varsigma / k}\right)\right]\right)(x) \\
& =\frac{\left(x^{s+1}-a^{s+1}\right)^{((v-\varsigma) / k)-1}}{(s+1)^{\varsigma / k}} S_{(l, m)}^{(\varsigma, v-\varsigma, \sigma, q, k)}\left(\tau\left(x^{s+1}-a^{s+1}\right)^{\varsigma / k}\right), \\
& \left({ }_{k}^{s} D_{a+}^{\varsigma, v}\left[\left(z^{s+1}-a^{s+1}\right)^{(v / k)-1} S_{(l, m)}^{(\varsigma, v, \sigma, q, k)}\left(\tau\left(z^{s+1}-a^{s+1}\right)^{\varsigma / k}\right)\right]\right)(x) \\
& =\frac{\left(x^{s+1}-a^{s+1}\right)^{((v-\varsigma) / k)-1}}{(s+1)^{-\varsigma / k}} S_{(l, m)}^{(\varsigma, v-\varsigma, \sigma, q, k)}\left(\tau\left(x^{s+1}-a^{s+1}\right)^{\varsigma / k}\right) \text {. }
\end{aligned}
$$

Corollary 16. Choosing $\xi=1$ in equations (38), (39), and (40) and using (14) yield

$$
\begin{aligned}
& \left({ }_{k}^{s} I_{a+}^{\varsigma}\left[\left(z^{s+1}-a^{s+1}\right)^{(v / k)-1} S_{(l, m)}^{(\zeta,, \sigma, 1, k)}\left(\tau\left(z^{s+1}-a^{s+1}\right)^{\varsigma / k}\right)\right]\right)(x) \\
& =\frac{\left(x^{s+1}-a^{s+1}\right)^{((v+\varsigma) / k)-1}}{(s+1)^{\zeta / k}} S_{(l, m)}^{(\varsigma, v+\varsigma, \sigma, k)}\left(\tau\left(x^{s+1}-a^{s+1}\right)^{\varsigma / k}\right), \\
& \left({ }_{k}^{s} D_{a+}^{\varsigma}\left[\left(z^{s+1}-a^{s+1}\right)^{(v / k)-1} S_{(l, m)}^{(\zeta, v, \sigma, 1, k)}\left(\tau\left(z^{s+1}-a^{s+1}\right)^{\varsigma / k}\right)\right]\right)(x) \\
& =\frac{\left(x^{s+1}-a^{s+1}\right)^{((v-\varsigma) / k)-1}}{(s+1)^{\zeta / k}} S_{(l, m)}^{(\zeta, v-\varsigma, \sigma, k)}\left(\tau\left(x^{s+1}-a^{s+1}\right)^{\varsigma / k}\right),
\end{aligned}
$$

$$
\begin{aligned}
& \left({ }_{k}^{s} D_{a+}^{\zeta, v}\left[\left(z^{s+1}-a^{s+1}\right)^{(v / k)-1} S_{(l, m)}^{(\zeta, v, \sigma, l, k)}\left(\tau\left(z^{s+1}-a^{s+1}\right)^{\zeta / k}\right)\right]\right)(x) \\
& =\frac{\left(x^{s+1}-a^{s+1}\right)^{((v-\varsigma) / k)-1}}{(s+1)^{-\zeta / k}} S_{(l, m)}^{(\zeta, v-\zeta, \sigma, k)}\left(\tau\left(x^{s+1}-a^{s+1}\right)^{\zeta / k}\right) .
\end{aligned}
$$

Corollary 17. Choosing $k=1$ in equations (49), (50), and (51) then S-function reduces to K-function, and using (16), it yields

$$
\begin{aligned}
& \left({ }^{s} I_{a^{\zeta}}^{\varsigma}\left[\left(z^{s+1}-a^{s+1}\right)^{\nu-1} S_{(l, m)}^{(\varsigma, v, \sigma, 1,1)}\left(\tau\left(z^{s+1}-a^{s+1}\right)^{\varsigma}\right)\right]\right)(x) \\
& =\frac{\left(x^{s+1}-a^{s+1}\right)^{v+\varsigma-1}}{(s+1)^{\varsigma}} K_{(l, m)}^{(\varsigma, v+\varsigma, \sigma)}\left(\tau\left(x^{s+1}-a^{s+1}\right)^{\varsigma}\right),
\end{aligned}
$$

$$
\begin{aligned}
& \left({ }^{s} D_{a+}^{\varsigma}\left[\left(z^{s+1}-a^{s+1}\right)^{v-1} S_{(l, m)}^{(\varsigma, v,, 1,1)}\left(\tau\left(z^{s+1}-a^{s+1}\right)^{\varsigma}\right)\right]\right)(x) \\
& =\frac{\left(x^{s+1}-a^{s+1}\right)^{v-\varsigma-1}}{(s+1)^{\varsigma}} K_{(l, m)}^{(\varsigma, v-\varsigma, \sigma)}\left(\tau\left(x^{s+1}-a^{s+1}\right)^{\varsigma}\right), \\
& \left({ }^{s} D_{a+}^{\varsigma, v}\left[\left(z^{s+1}-a^{s+1}\right)^{v-1} S_{(l, m)}^{(\zeta, v, \sigma, 1,1)}\left(\tau\left(z^{s+1}-a^{s+1}\right)^{\varsigma}\right)\right]\right)(x) \\
& =\frac{\left(x^{s+1}-a^{s+1}\right)^{v-\varsigma-1}}{(s+1)^{-\varsigma}} K_{(l, m)}^{(\varsigma, v-\varsigma, \sigma)}\left(\tau\left(x^{s+1}-a^{s+1}\right)^{\varsigma}\right) .
\end{aligned}
$$

Corollary 18. Choosing $\sigma=1$ in equations (52), (53), and (54) then S-function becomes M-series defined by [32], and using (16), it yields

$$
\begin{aligned}
& \left({ }^{s} I_{a^{\varsigma}}^{\varsigma}\left[\left(z^{s+1}-a^{s+1}\right)^{v-1} S_{(l, m)}^{(\zeta, v, 1,1,1)}\left(\tau\left(z^{s+1}-a^{s+1}\right)^{\varsigma}\right)\right]\right)(x) \\
& =\frac{\left(x^{s+1}-a^{s+1}\right)^{v+\varsigma-1}}{(s+1)^{\varsigma}} M_{(l, m)}^{(\varsigma, v+\varsigma)}\left(\tau\left(x^{s+1}-a^{s+1}\right)^{\varsigma}\right)
\end{aligned}
$$

$$
\begin{aligned}
& \left({ }^{s} D_{a+}^{\varsigma}\left[\left(z^{s+1}-a^{s+1}\right)^{v-1} S_{(l, m)}^{(\varsigma, v, l, 1,1)}\left(\tau\left(z^{s+1}-a^{s+1}\right)^{\varsigma}\right)\right]\right)(x) \\
& =\frac{\left(x^{s+1}-a^{s+1}\right)^{v-\varsigma-1}}{(s+1)^{\varsigma}} M_{(l, m)}^{(\varsigma, v-\varsigma)}\left(\tau\left(x^{s+1}-a^{s+1}\right)^{\varsigma}\right), \\
& \left({ }^{s} D_{a+}^{\varsigma, v}\left[\left(z^{s+1}-a^{s+1}\right)^{v-1} S_{(l, m)}^{(\zeta, v, 1,1,1)}\left(\tau\left(z^{s+1}-a^{s+1}\right)^{\varsigma}\right)\right]\right)(x) \\
& =\frac{\left(x^{s+1}-a^{s+1}\right)^{v-\varsigma-1}}{(s+1)^{-\varsigma}} M_{(l, m)}^{(\varsigma, v-\varsigma)}\left(\tau\left(x^{s+1}-a^{s+1}\right)^{\varsigma}\right) .
\end{aligned}
$$

Corollary 19. Choosing $p=q=0$ in equations (55), (56), and (57) then S-function turns into generalized Mittag-Leffler function defined by Mittag-Leffler [34], and using (16), it yields

$$
\begin{aligned}
& \left({ }^{s} I_{a+}^{\varsigma}\left[\left(z^{s+1}-a^{s+1}\right)^{v-1} S_{(0,0)}^{(\varsigma, v, 1,1,1)}\left(\tau\left(z^{s+1}-a^{s+1}\right)^{\varsigma}\right)\right]\right)(x) \\
& =\frac{\left(x^{s+1}-a^{s+1}\right)^{v+\varsigma-1}}{(s+1)^{\varsigma}} E_{(\varsigma, v+\varsigma)}\left(\tau\left(x^{s+1}-a^{s+1}\right)^{\varsigma}\right), \\
& \left({ }^{s} D_{a+}^{\varsigma}\left[\left(z^{s+1}-a^{s+1}\right)^{v-1} S_{(0,0)}^{(\varsigma, v, 1,1,1)}\left(\tau\left(z^{s+1}-a^{s+1}\right)^{\varsigma}\right)\right]\right)(x) \\
& =\frac{\left(x^{s+1}-a^{s+1}\right)^{v-\varsigma-1}}{(s+1)^{\varsigma}} E_{(\varsigma, v-\varsigma)}\left(\tau\left(x^{s+1}-a^{s+1}\right)^{\varsigma}\right), \\
& \left({ }^{s} D_{a+}^{\varsigma, v}\left[\left(z^{s+1}-a^{s+1}\right)^{v-1} S_{(0,0)}^{(\varsigma, v, 1,1,1)}\left(\tau\left(z^{s+1}-a^{s+1}\right)^{\varsigma}\right)\right]\right)(x) \\
& =\frac{\left(x^{s+1}-a^{s+1}\right)^{v-\varsigma-1}}{(s+1)^{-\varsigma}} E_{(\varsigma, v-\varsigma)}\left(\tau\left(x^{s+1}-a^{s+1}\right)^{\varsigma}\right) .
\end{aligned}
$$


If we substitute $s=0$ in (38), (39), and (40), then we have some special results of the S-function.

Corollary 20. Suppose $k>0, x>a\left(a \in \mathfrak{R}_{+}=[0, \infty)\right)$ and $\varsigma, v$ , $\sigma, \tau \in \mathbb{C}, \mathfrak{R}(v), \mathfrak{R}(\varsigma)>0, \mathfrak{R}(\sigma)>0, \mathfrak{R}(\varsigma)>0$, then

$$
\begin{aligned}
& \left({ }_{k} I_{a+}^{\varsigma}\left[(t-a)^{(v / k)-1} S_{(l, m)}^{(\zeta, v, \xi, k)}\left(\tau(t-a)^{\varsigma / k}\right)\right]\right)(x) \\
& =(x-a)^{((v+\varsigma) / k)-1} S_{(l, m)}^{(\varsigma, v+\zeta, \sigma, \xi, k)}\left(\tau(x-a)^{\varsigma / k}\right) \text {, } \\
& \left({ }_{k} D_{a+}^{\varsigma}\left[(t-a)^{(v / k)-1} S_{(l, m)}^{(\zeta, v, \sigma, \xi, k)}\left(\tau(t-a)^{\varsigma / k}\right)\right]\right)(x) \\
& =(x-a)^{((v-\zeta) / k)-1} S_{(l, m)}^{(\zeta, v-\zeta, \xi, \xi, k)}\left(\tau(x-a)^{\varsigma / k}\right), \\
& \left({ }_{k} D_{a+}^{\zeta, v}\left[(t-a)^{(v / k)-1} S_{(l, m)}^{(\zeta, v, \sigma, \xi, k)}\left(\tau(t-a)^{\zeta / k}\right)\right]\right)(x) \\
& =(x-a)^{((v-\zeta) / k)-1} S_{(l, m)}^{(\zeta, v-\zeta, \sigma, \xi, k)}\left(\tau(x-a)^{\zeta / k}\right) \text {. }
\end{aligned}
$$

If we substitute $k=1$ in (59), (60), and (61), then we have some special results of the S-function.

Corollary 21. Suppose $x>a\left(a \in \Re_{+}=[0, \infty)\right)$ and $\varsigma, v, \sigma, \tau$ $\in \mathbb{C}, \mathfrak{R}(v), \mathfrak{R}(\varsigma)>0, \mathfrak{R}(\sigma)>0, \mathfrak{R}(\varsigma)>0$, then

$$
\begin{aligned}
& \left(I_{a+}^{\varsigma}\left[(t-a)^{\nu-1} S_{(l, m)}^{(\varsigma, v, \sigma, \xi, 1)}\left(\tau(t-a)^{\varsigma}\right)\right]\right)(x) \\
& =(x-a)^{v+\varsigma-1} S_{(l, m)}^{(\zeta, v+\zeta, \sigma, \xi, 1)}\left(\tau(x-a)^{\varsigma}\right), \\
& \left(D_{a+}^{\varsigma}\left[(t-a)^{v-1} S_{(l, m)}^{(\varsigma, v, \sigma, \xi, 1)}\left(\tau(t-a)^{\varsigma}\right)\right]\right)(x) \\
& =(x-a)^{\nu-\varsigma-1} S_{(l, m)}^{(\zeta, v-\zeta, \sigma, \xi, 1)}\left(\tau(x-a)^{\varsigma}\right), \\
& \left(D_{a+}^{\varsigma, v}\left[(t-a)^{\nu-1} S_{(l, m)}^{(\varsigma,, \sigma, \xi, k)}\left(\tau(t-a)^{\varsigma}\right)\right]\right)(x) \\
& =(x-a)^{v-\varsigma-1} S_{(l, m)}^{(\varsigma, v-\varsigma, \sigma, \xi, 1)}\left(\tau(x-a)^{\varsigma}\right) .
\end{aligned}
$$

\section{Some Properties of the Operator $\left({ }_{k}^{s} \varepsilon_{a+; \zeta, v}^{\tau ; \sigma} f\right)(x)$}

Theorem 22. For $k>0$ and $\varsigma, v, \sigma, \xi, \zeta, \tau \in \mathbb{C}, \mathfrak{R}(\varsigma)>0, \mathfrak{R}$ $(v)>0, \mathfrak{R}(\sigma)>0$ and $\mathfrak{R}(\varsigma)>0$, we have

$$
\begin{aligned}
& \left({ }_{k}^{s} \varepsilon_{a+; \zeta, v}^{\tau ; \xi \xi}\left[z^{s+1}-a^{s+1}\right]^{\varsigma / k}\right)(x)=\frac{\left(x^{s+1}-a^{s+1}\right)^{((\varsigma+v) / k)-1} \Gamma_{k}(\varsigma)}{(s+1)} \\
& \cdot S_{(l, m)}^{(\varsigma, v+\zeta, \sigma, \xi, k)}\left(\tau\left(x^{s+1}-a^{s+1}\right)^{\varsigma / k}\right) .
\end{aligned}
$$

Proof. From (20),

$$
\begin{aligned}
\left({ }_{k}^{s} \varepsilon_{a+; \zeta, \gamma}^{\tau ; \sigma, \xi} f\right)(x)= & \frac{1}{k} \int_{a}^{x}\left(x^{s+1}-z^{s+1}\right)^{(v / k)-1} S_{(l, m)}^{(\varsigma, v, \sigma, \xi, k)} \\
& \cdot\left(\tau\left(x^{s+1}-z^{s+1}\right)^{\varsigma / k}\right) z^{s} f(z) d z .
\end{aligned}
$$

Therefore, we have

$$
\begin{aligned}
& \left({ }_{k}^{s} \varepsilon_{a+; \zeta, v}^{\tau ; \sigma, \xi}\left[z^{s+1}-a^{s+1}\right]^{\zeta / k}\right)(x)=\frac{1}{k} \int_{a}^{x}\left(x^{s+1}-z^{s+1}\right)^{(v / k)-1} \\
& \cdot\left(z^{s+1}-a^{s+1}\right)^{(\varsigma / k)-1} S_{(l, m)}^{(\varsigma, v, \xi, \xi, k)}\left(\tau\left(x^{s+1}-z^{s+1}\right)^{\varsigma / k}\right) z^{s} d z \\
& =\sum_{n=0}^{\infty} \frac{\left(p_{1}\right)_{n} \cdots\left(p_{l}\right)_{n}(\sigma)_{n \xi, t} \tau^{n}}{\left(q_{1}\right)_{n} \cdots\left(q_{m}\right)_{n} \Gamma_{k}(n \varsigma+v) n !} \\
& \text {. }\left(\frac{1}{k} \int_{a}^{x}\left(z^{s+1}-a^{s+1}\right)^{(\varsigma / k)-1}\left(x^{s+1}-z^{s+1}\right)^{((v+\varsigma n) / k)-1} z^{s} d z\right) \\
& =\sum_{n=0}^{\infty} \frac{\left(a_{1}\right)_{n} \cdots\left(a_{p}\right)_{n}(\sigma)_{n \xi, k} \tau^{n}}{\left(q_{1}\right)_{n} \cdots\left(q_{m}\right)_{n}(s+1) \Gamma_{k}(n \varsigma+v) n !} \\
& \cdot\left(x^{s+1}-a^{s+1}\right)^{((v+\varsigma+\varsigma n) / k)-1} B_{k}(v+\varsigma n, \varsigma) \\
& =\sum_{n=0}^{\infty} \frac{\left(a_{1}\right)_{n} \cdots\left(a_{p}\right)_{n}(\sigma)_{n \xi, k} \tau^{n}\left(x^{s+1}-a^{s+1}\right)^{((v+\zeta+\zeta n) / k)-1}}{\left(q_{1}\right)_{n} \cdots\left(q_{m}\right)_{n}(s+1) \Gamma_{k}(n \varsigma+v) n !} \\
& \cdot \frac{\Gamma_{k}(v+\varsigma n) \Gamma_{k}(\varsigma)}{\Gamma_{k}(v+\varsigma+\varsigma n)}=\frac{\left(x^{s+1}-a^{s+1}\right)^{((v+\varsigma) / k)-1} \Gamma_{k}(\varsigma)}{(s+1)} \\
& \cdot S_{(l, m)}^{(\varsigma, v+\varsigma, \sigma, \xi, k)}\left(\tau\left(x^{s+1}-a^{s+1}\right)^{\varsigma / k}\right) \text {, }
\end{aligned}
$$

which completes the desired proof.

Theorem 23. Suppose let $f \in L_{1}[a, b], s \in \mathfrak{R} \backslash[-1], k>0$ and $\varsigma, \nu, \sigma, \xi, \zeta, \tau \in \mathbb{C}, \mathfrak{R}(\varsigma)>0, \mathfrak{R}(v)>0, \mathfrak{R}(\sigma)>0$ and $\mathfrak{R}(\varsigma)>$ 0 , then ${ }_{k}^{s} \varepsilon_{a+; s, v}^{\tau ; \sigma, \xi} f(x)$ exit for any $x \in[a, b]$.

Proof. Assume that $\nabla=[a, b] \times[a, b]$ and $U: \nabla \longrightarrow \Re$ such that $U(x, t)=\left[\left(x^{s+1}-z^{s+1}\right) z^{s}\right]$ for all $x \in[a, b]$. It is obvious that $U=U_{+}+U_{-}$where

$$
\begin{aligned}
& U_{+}(x, t)=\left(\begin{array}{ll}
\left(x^{s+1}-z^{s+1}\right)^{(v / k)-1} z^{s}, & a \leq t \leq x \leq b . \\
0, & a \leq x \leq t \leq b .
\end{array}\right. \\
& U_{-}(x, t)=\left(\begin{array}{ll}
\left(z^{s+1}-x^{s+1}\right)^{(v / k)-1} x^{s}, & a \leq t \leq x \leq b . \\
0, & a \leq x \leq t \leq b .
\end{array}\right.
\end{aligned}
$$

As $U$ is measurable on $\zeta$, we can write

$$
\begin{aligned}
\int_{a}^{b} U(x, z) d z & =\int_{a}^{x} U(x, z) d z=\int_{a}^{x}\left(x^{s+1}-z^{s+1}\right)^{(v / k)-1} z^{s} d z \\
& =\frac{k}{v}\left(x^{s+1}-z^{s+1}\right)^{v / k}
\end{aligned}
$$

So, we get

$$
\begin{aligned}
& =\sum_{n=0}^{\infty} \frac{\left(p_{1}\right)_{n} \cdots\left(p_{l}\right)_{n}(\sigma)_{n \xi, k} \tau^{n}}{\left(q_{1}\right)_{n} \cdots\left(q_{m}\right)_{n} \Gamma_{k}(n \varsigma+v) n !}\left(\int_{a}^{x}\left(x^{s+1}-z^{s+1}\right)^{((v+\varsigma n) / k)-1} z^{s} d z\right) \\
& =\sum_{n=0}^{\infty} \frac{\left(a_{1}\right)_{n} \cdots\left(a_{p}\right)_{n}(\sigma)_{n \xi, k}\left(\tau\left(x^{s+1}-z^{s+1}\right)^{\varsigma / k}\right)^{n}}{\left(q_{1}\right)_{n} \cdots\left(q_{m}\right)_{n} \Gamma_{k}(n \varsigma+v) n !} \frac{k}{v+\varsigma n}\left(x^{s+1}-a^{s+1}\right)^{v / k} .
\end{aligned}
$$


Again, integral the above term, we have

$$
\begin{aligned}
\int_{a}^{b} & \left(\int_{a}^{b} U(x, t) S_{(l, m)}^{(\varsigma, v, \sigma, \xi, k)}\left(\tau\left(x^{s+1}-z^{s+1}\right)^{\varsigma / k}\right)|f(x)| d z\right) d x \\
= & \int_{a}^{b}|f(x)|\left(\int_{a}^{x} U(x, z) S_{(l, m)}^{(\varsigma, v, \sigma, \xi, k)}\left(\tau\left(x^{s+1}-z^{s+1}\right)^{\varsigma / k}\right) d z\right) d x \\
= & \sum_{n=0}^{\infty} \frac{\left(a_{1}\right)_{n} \cdots\left(a_{p}\right)_{n}(\sigma)_{n \xi, k} \tau^{n}}{\left(q_{1}\right)_{n} \cdots\left(q_{m}\right)_{n} \Gamma_{k}(n \varsigma+v) n !} \frac{k}{v+\varsigma n} \\
& \cdot\left(\int_{a}^{b}\left(x^{s+1}-a^{s+1}\right)^{(v+\varsigma n) / k}|f(x)| d x\right) \\
\leq & \sum_{n=0}^{\infty} \frac{\left(a_{1}\right)_{n} \cdots\left(a_{p}\right)_{n}(\sigma)_{n \xi, k}\left(\tau\left(b^{s+1}-a^{s+1}\right)^{\varsigma / k}\right)^{n}}{\left(q_{1}\right)_{n} \cdots\left(q_{m}\right)_{n} \Gamma_{k}(n \varsigma+v) n !} \\
& \cdot \frac{k^{2}}{(v+\varsigma n)(v+\varsigma n+k)}\left(b^{s+1}-a^{s+1}\right)^{(v / k)+1} \\
& \times \int_{a}^{b}|f(x)| d x \leq\left(b^{s+1}-a^{s+1}\right)^{(v / k)+1} \\
& \cdot \sum_{n=0}^{\infty} \frac{\left(a_{1}\right)_{n} \cdots\left(a_{p}\right)_{n}(\sigma)_{n \xi, k}\left(\tau\left(b^{s+1}-a^{s+1}\right)^{\varsigma / k}\right)^{n}}{\left(q_{1}\right)_{n} \cdots\left(q_{m}\right)_{n} \Gamma_{k}(n \varsigma+v) n !} \\
& \times \frac{k^{2}}{(v+\varsigma n)(v+\varsigma n+k)^{\prime}}\|f\|_{1} \leq \infty .
\end{aligned}
$$

The $V: \zeta \longrightarrow \Re$ function is then such that $V(x, t)=U$ $(x, t) f(x)$ can be integrated into $\zeta$ by Toneli's theorem. Thus, by Fubini's theorem $\int_{a}^{b} U(x, t) S_{(l, m)}^{(\varsigma, v, \sigma, \xi, k)}\left(\tau\left(x^{s+1}-z^{s+1}\right)^{\varsigma / k}\right)$ $f(x) d x$ is an integrable function on $[a, b]$, as a function of $t$ $\in[a, b]$. Thus, ${ }_{k}^{s} \varepsilon_{a+; \zeta, v}^{\tau ; \sigma, \xi} f(x)$ exists.

Theorem 24. For $\varsigma \in \mathbb{C}, \varsigma, v, \sigma, \xi, \zeta, \tau \in \mathbb{C}, \mathfrak{R}(\varsigma)>0, \mathfrak{R}(v)>$ $0, \mathfrak{R}(\sigma)>0$ and $\mathfrak{R}(\varsigma)>0, k>0, s \in \mathfrak{R} \backslash\{-1\}$ and $x>a$, the following result holds:

$$
\begin{aligned}
\left({ }_{k}^{s} I_{a+}^{\zeta}\left[{ }_{k}^{s} \varepsilon_{a+; \zeta, v}^{\tau ; \sigma, \xi} f\right]\right)(x) & =\frac{1}{(s+1)^{\varsigma / k}}\left({ }_{k}^{s} \varepsilon_{a+; \zeta, v+\zeta}^{\tau ; \sigma, \xi} f\right)(x) \\
& =\left({ }_{k}^{s} \varepsilon_{a+; \zeta, v+\varsigma}^{\tau ; \sigma, \xi}\left[{ }_{k}^{s} I_{a+}^{\varsigma} f\right]\right)(x),
\end{aligned}
$$

for any $f \in L(\varsigma, v)$.

Proof. From (20) and (24), we observe

$$
\begin{gathered}
\left({ }_{k}^{s} I_{a+}^{\varsigma}\left[{ }_{k}^{s} \varepsilon_{a+; \zeta, v}^{\tau ; \sigma, \xi} f\right]\right)(x)=\frac{(s+1)^{1-(\varsigma / k)}}{k \Gamma_{k}(\varsigma)} \int_{a}^{x} \frac{\left[{ }_{k}^{s} \varepsilon_{a+; \zeta, v}^{\tau ; \sigma, \xi} f(z)\right]}{\left(x^{s+1}-z^{s+1}\right)^{1-(\varsigma / k)}} z^{s} d z \\
=\frac{(s+1)^{1-(\varsigma / k)}}{k^{2} \Gamma_{k}(\varsigma)} \int_{a}^{x}\left(x^{s+1}-z^{s+1}\right)^{(\varsigma / k)-1} \times\left[\int_{a}^{z}\left(z^{s+1}-u^{s+1}\right)^{(v / k)-1}\right. \\
\left.\cdot S_{(l, m)}^{(\varsigma, v, \sigma, \xi, k)}\left(\tau\left(x^{s+1}-z^{s+1}\right)^{\varsigma / k}\right) f(u) u^{s} d u\right] z^{s} d z .
\end{gathered}
$$

By changing the order of integration, we obtain

$$
\begin{aligned}
& \left({ }_{k}^{s} I_{a+}^{\varsigma}\left[{ }_{k}^{s} \varepsilon_{a+; \zeta, v}^{\tau ; \sigma, \xi} f\right]\right)(x)=\frac{1}{k} \int_{a}^{x}\left[\frac{(s+1)^{1-(\varsigma / k)}}{k \Gamma_{k}(\varsigma)} \int_{u}^{x}\left(x^{s+1}-z^{s+1}\right)^{\varsigma / k}\right. \\
& \left.\cdot\left(z^{s+1}-u^{s+1}\right)^{(v / k)-1} S_{(l, m)}^{(\varsigma, v, \sigma, \xi, k)}\left(\tau\left(x^{s+1}-z^{s+1}\right)^{\varsigma / k}\right) z^{s} d z\right] u^{s} f(u) d u .
\end{aligned}
$$

Using (38), we have

$$
\begin{aligned}
& \left({ }_{k}^{s} I_{a+}^{\varsigma}\left[{ }_{k}^{s} \varepsilon_{a+; \zeta, v}^{\tau ; \sigma, \xi} f\right]\right)(x)=\frac{1}{k(s+1)^{\varsigma / k}} \int_{a}^{x}\left(x^{s+1}-z^{s+1}\right)^{((v+\varsigma) / k)-1} \\
& \times S_{(l, m)}^{(\varsigma, v+\varsigma, \sigma, \xi, k)}\left(\tau\left(x^{s+1}-z^{s+1}\right)^{\varsigma / k}\right) u^{s} f(u) d u .
\end{aligned}
$$

Thus, we get

$$
\left({ }_{k}^{s} I_{a+}^{\varsigma}\left[{ }_{k}^{s} \varepsilon_{a+; \zeta, v}^{\tau ; \sigma, \xi} f\right]\right)(x)=\frac{1}{(s+1)^{\varsigma / k}}\left({ }_{k}^{s} \varepsilon_{a+; \zeta, v+\zeta}^{\tau ; \sigma, \xi} f\right)(x) .
$$

To prove the second part of the theorem, consider the RHS of (70); then, by applying (20), we get

$$
\begin{gathered}
\left({ }_{k}^{s} I_{a+}^{\varsigma}\left[{ }_{k}^{s} \varepsilon_{a+;, \zeta, v}^{\tau ; \sigma, \xi} f\right]\right)(x)=\frac{1}{k} \int_{a}^{x}\left(x^{s+1}-z^{s+1}\right)^{(v / k)-1} S_{(l, m)}^{(\varsigma, v, \sigma, \xi, k)} \\
\cdot\left(\tau\left(x^{s+1}-z^{s+1}\right)^{\varsigma / k}\right)\left[{ }_{k}^{s} I_{a+}^{\varsigma} f\right](z) z^{s} d z=\frac{1}{k} \int_{a}^{x}\left(x^{s+1}-z^{s+1}\right)^{(v / k)-1} S_{(l, m)}^{(\varsigma, v, \sigma, \xi, k)} \\
\cdot\left(\tau\left(x^{s+1}-z^{s+1}\right)^{\varsigma / k}\right) \times\left(\frac{(s+1)^{1-(\varsigma / k)}}{k \Gamma_{k}(\varsigma)} \int_{a}^{z} \frac{f(u)}{\left(z^{s+1}-u^{s+1}\right)^{1-(\varsigma / k)} u^{s} d u}\right) d z .
\end{gathered}
$$

By interchanging the order of integration, we have

$$
\begin{aligned}
& \left({ }_{k}^{s} I_{a+}^{\varsigma}\left[{ }_{k}^{s} \varepsilon_{a+; \zeta, v}^{\tau ; \sigma, \xi} f\right]\right)(x)=\frac{1}{k} \int_{a}^{x} \frac{(s+1)^{1-(\varsigma / k)}}{k \Gamma_{k}(\varsigma)} \\
& \quad \times\left[\int_{u}^{x}\left(x^{s+1}-z^{s+1}\right)^{(v / k)-1}\left(z^{s+1}-u^{s+1}\right)^{(\varsigma / k)-1} S_{(l, m)}^{(\varsigma, v, \sigma, \xi, k)}\right. \\
& \left.\cdot\left(\tau\left(x^{s+1}-z^{s+1}\right)^{\varsigma / k}\right) z^{s} d z\right] \times f(u) u^{s} d u
\end{aligned}
$$

Again, by making use of (24) and using (38), we get

$$
\left({ }_{k}^{s} I_{a+}^{\varsigma}\left[{ }_{k}^{s} \varepsilon_{a+; \zeta, v}^{\tau ; \sigma, \xi} f\right]\right)(x)=\frac{1}{(s+1)^{\zeta / k}}\left({ }_{k}^{s} \varepsilon_{a+; \zeta, v+\varsigma}^{\tau ; \sigma, \xi} f\right)(x) .
$$

Thus, (74) and (77) complete the proof of (70).

\section{Concluding Remarks}

In this article, we develop some new results of $(k, s)$-fractional integral and differential operators involving $S$-function under the extension of left and right R-L $(k, s)$ -fractional integral and differential operators. Also, we find 
some special cases of functions like $k$-Mittag-Leffler function, $K$-function, and $M$-series. If we set $l=0=m$, then we obtained results of the generalized $k$-Mittag-Leffler function given by [37], and if we set $s=0$, the results obtained are reduced to the well-known results given by [38]. If we select $k=1$ and $s=0$, then the results obtained are reduced to the Mittag-Leffler function's well-known results.

\section{Data Availability}

No data were used to support this study.

\section{Conflicts of Interest}

There is no conflict of interest regarding the publication of this article.

\section{References}

[1] V. Kiryakova, Generalized Fractional Calculus and Applications, vol. 38, Longman Scientific and Technical, Harlow, 1994.

[2] K. S. Miller and B. Ross, An Introduction to the Fractional Calculus and Fractional Differential Equations, Wiley, New York, 1993.

[3] R. S. Adiguzel, U. Aksoy, E. Karapinar, and I. M. Erhan, "On the solutions of fractional differential equations via Geraghty type hybrid contractions," Applied and Computational Mathematics, vol. 20, no. 2, pp. 313-333, 2021.

[4] A. A. Kilbas, M. Saigo, and R. K. Saxena, "Generalized MittagLeffler function and generalized fractional calculus operators," Integral Transforms and Special Functions, vol. 15, no. 1, pp. 31-49, 2004.

[5] S. D. Purohit and S. L. Kalla, "On fractional partial differential equations related to quantum mechanics," Journal of Physics A: Mathematical and Theoretical, vol. 44, no. 4, article 045202, 2011.

[6] R. Sevinik-Adıgüzel, Ü. Aksoy, E. Karapınar, and I. M. Erhan, "Uniqueness of solution for higher-order nonlinear fractional differential equations with multi-point and integral boundary conditions," Revista de la Real Academia de Ciencias Exactas, Físicas y Naturales. Serie A. Matemáticas, vol. 115, pp. 1-16, 2021.

[7] H. M. Srivastava and Ž. Tomovski, "Fractional calculus with an integral operator containing a generalized Mittag-Leffler function in the kernel," Applied Mathematics and Computation, vol. 211, pp. 198-210, 2009.

[8] D. Foukrach, S. Bouriah, M. Benchohra, and E. Karapinar, "Some new results for $\$ \$ \backslash p s i \quad-\$$ Hilfer fractional pantograph-type differential equation depending on $\$ \$ \backslash p s i$ -\$\$Riemann-Liouville integral," Journal of Analysis, pp. 125,2021

[9] B. Alqahtani, A. Fulga, F. Jarad, and E. Karapınar, "Nonlinear _F_-contractions on _b_- -metric spaces and differential equations in the frame of fractional derivatives with Mittag-Leffler kernel," Chaos, Solitons \& Fractals, vol. 128, pp. 349-354, 2019.

[10] E. Karapina, H. D. Binh, N. H. Luc, and N. H. Can, "On continuity of the fractional derivative of the time-fractional semilinear pseudo-parabolic systems," Advances in Differential Equations, vol. 2021, no. 1, pp. 1-24, 2021.
[11] D. S. Mitrinovic, J. E. Pecaric, and A. M. Fink, "Classical and new inequalities in analysis," in Mathematics and Its Applications, Springer, Netherlands, 1993.

[12] A. G. Garcia, F. Marcellhn, and L. Salto, "A distributional study of discrete classical orthogonal polynomials," Journal of Computational and Applied Mathematics, vol. 57, no. 1-2, pp. 147-162, 1995.

[13] L. M. Milne-Thomson, The Calculus of Finite Differences, Macmillan \& Co., London, 1993.

[14] R. Diaz and E. Pariguan, "On hypergeometric functions and Pochhammer $k$-symbol," Divulgaciones Mathematicas, vol. 15, no. 2, pp. 179-192, 2007.

[15] S. Mubeen and G. M. Habibullah, " $k$-Fractional integrals and application," International Journal of Contemporary Mathematical Sciences, vol. 7, pp. 89-94, 2012.

[16] M. Z. Sarikaya, Z. Dahmani, M. E. Kiris, and F. Ahmad, “ $(k ; s)$ -Riemann-Liouville fractional integral and applications," Hacettepe Journal of Mathematics and Statistics, vol. 45, pp. 77-89, 2016.

[17] R. M. Evans and U. N. Katugampola, "Applications of fractional calculus in solving Abel-type integral equations: surface-volume reaction problem," Computers \& Mathematcs with Applications, vol. 73, no. 6, pp. 1346-1362, 2017.

[18] J. A. T. Machado, M. F. Silva, R. S. Barbosa et al., "Some applications of fractional calculus in engineering," Mathematical Problems in Engineering, vol. 2010, no. 34, 2010.

[19] P. Agarwal, M. Jaleli, and M. Tomar, "Certain HermiteHadamard type inequalities via generalized $k$-fractional integrals," Journal of inequalities and applications, vol. 2017, no. 1,2017

[20] M. Aldhaifallah, M. Tomar, K. S. Nisar, and S. D. Purohit, "Some new inequalities for $(k, s)$-fractional integrals," Journal of Nonlinear Sciences and Applications, vol. 9, no. 9, pp. 5374-5381, 2016.

[21] S. Mubeen and S. Iqbal, "Grüss type integral inequalities for generalized Riemann-Liouville $k$-fractional integrals," Journal of Inequalities and Applications, vol. 2016, 13 pages, 2016.

[22] M. Priya and R. Uthayakumar, "Analytical properties of $(k, s)$ Riemann-Liouville fractional integral and its fractal dimension," Journal of Analysis, pp. 1-12, 2021.

[23] M. Tomar, S. Maden, and E. Set, “\$\$\{|varvec $\{(\mathrm{k}, \mathrm{s})\}\} \$ \$(\mathrm{k}, \mathrm{s})$ -Riemann-Liouville fractional integral inequalities for continuous random variables," Arabian Journal of Mathematics, vol. 6, no. 1, pp. 55-63, 2017.

[24] R. K. Saxena and J. Daiya, "Integral transforms of the $S$-functions," Le Mathematiche, pp. 147-157, 2015.

[25] H. Amsalu, B. Shimelis, and D. L. Suthar, "Pathway fractional integral formulas involving S-function in the kernel," Mathematical Problems in Engineering, vol. 2020, Article ID 4236823, 6 pages, 2020.

[26] D. L. Suthar, "Certain fractional integral operators pertaining to S-function," Cogent Mathematics \& Statistics, vol. 7, no. 1, article 1781506, 2020.

[27] D. L. Suthar and H. Amsalu, "Fractional integral and derivative formulas by using Marichev-Saigo-Maeda operators involving the S-function," Abstract and Applied Analysis, vol. 2019, Article ID 6487687, 19 pages, 2019.

[28] D. L. Suthar and T. Hailay, "Euler-type integral operator involving S-function," Abstract and Applied Analysis, vol. 2020, 7 pages, 2020. 
[29] H. Tadesse, H. Habenom, A. Alaria, and B. Shimelis, "Composition formulae for the $k$-fractional calculus operator with the $S$ -function," Journal of Mathematics, vol. 2021, Article ID 7379820, 12 pages, 2021.

[30] R. K. Saxena, J. Daiya, and A. Singh, "Integral transforms of the $k$-generalized Mittag-Leffler function $E_{k, \alpha, \beta}^{\gamma, \tau}(z)$," Le Matematiche, vol. 69, no. 2, pp. 7-16, 2014.

[31] K. Sharma, "Application of fractional calculus operators to related areas," General Mathematics Notes, vol. 7, no. 1, pp. 33-40, 2011.

[32] K. Sharma and R. Jain, "A note on a generalized $M$-series as a special function of fractional calculus," Fractional Calculus and Applied Analysis, vol. 12, no. 4, pp. 449-452, 2009.

[33] D. L. Suthar, F. Gidaf, and M. Andualem, "Certain properties of generalized $M$-series under generalized fractional integral operators," Journal of Mathematics, vol. 2021, Article ID 5527819, 10 pages, 2021.

[34] G. Mittag-Leffler, "Sur la représentation analytique d'une branche uniforme d'une fonction monogène: cinquième note," Acta Mathematica, vol. 29, pp. 101-181, 1905.

[35] A. A. Kilbas, H. M. Srivastava, and J. J. Trujillo, "Theory and applications of fractional differential equations," in North-Holland Mathematics Studies, vol. 204, Elsevier, Amsterdam, 2006.

[36] S. G. Samko, A. A. Kilbas, and O. I. Marichev, Fractional Integrals and Derivatives: Theory and Applications, Gordon and Breach Science Publishers, Switzerland, 1993.

[37] K. S. Nisar, G. Rahman, D. Baleanu, S. Mubeen, and M. Arshad, "The $(k, s)$-fractional calculus of $k$-Mittag-Leffler function," Advances in Difference Equations, vol. 2017, no. 1, 2017.

[38] G. A. Dorrego, "Generalized Riemann-Liouville fractional operators associated with a generalization of the Prabhakar integral operator," Progress in Fractional Differentiation and Applications, vol. 2, no. 2, pp. 131-140, 2016. 